Review

\title{
Development of Delpazolid for the Treatment of Tuberculosis
}

\author{
Young Lag Cho ${ }^{1}$ and Jichan Jang ${ }^{2, *}$ \\ 1 LegoChem Biosciences, Inc., 8-26 Munoyeongseo-ro, Daedeok-gu, Daejeon 34302, Korea; \\ young@legochembio.com \\ 2 Molecular Mechanisms of Antibiotics, Division of Life Science, Research Institute of Life Sciences, \\ Gyeongsang National University, Jinju 52828, Korea \\ * Correspondence: jichanjang@gnu.ac.kr; Tel.: +82-(0)55-772-1368
}

Received: 3 March 2020; Accepted: 23 March 2020; Published: 25 March 2020

\begin{abstract}
A novel oxazolidinone with cyclic amidrazone, delpazolid (LCB01-0371), was synthesized by LegoChem BioSciences, Inc. (Daejeon, Korea). Delpazolid can improve the minimum bactericidal concentration of Mycobacterium tuberculosis H37Rv and significantly reduce resistance rates, especially of multi-drug-resistant tuberculosis (MDR-TB) isolates, compared with linezolid. Therefore, delpazolid can be used to treat MDR-TB. The safety, tolerability, and pharmacokinetics of delpazolid have been evaluated in a phase 1 clinical trial, which revealed that it does not cause adverse events such as myelosuppression even after three weeks of repeated dosing. Interim efficacy and safety results, particularly those from a clinical phase 2a early bactericidal activity trial including patients with drug-susceptible tuberculosis, were reported and the findings will be further analyzed to guide phase 2a studies.
\end{abstract}

Keywords: Mycobacterium tuberculosis; delpazolid; drug discovery; multi-drug resistance

\section{Linezolid, the First Oxazolidinone Antibacterial Agent}

Oxazolidinone is a heterocyclic organic compound containing both nitrogen and oxygen in a 5-membered ring and is mainly used as an antimicrobial agent. This class of antimicrobials is active against a large spectrum of Gram-positive bacteria, including methicillin-resistant Staphylococcus aureus (MRSA), vancomycin-resistant enterococci (VRE), vancomycin-intermediate strains, and penicillin-resistant pneumococci, and acts via inhibiting protein synthesis [1,2].

Linezolid is the first oxazolidinone antimicrobial to be developed; it exhibits a high degree of in vitro activity against various Gram-positive pathogens [3]. Linezolid exhibits bactericidal activity against Mycobacterium tuberculosis and has been used to treat rifampicin-resistant tuberculosis (RR-TB) or multi-drug-resistant tuberculosis (MDR-TB) [4]. Although the integration of linezolid into RR-TB or MDR-TB treatment can improve outcomes, prolonged administration is often limited by long-term side effects, including reversible myelosuppression, potentially irreversible optic neuropathy, and peripheral neuropathy [5]. Therefore, safety and tolerability are critical issues to consider when prescribing these antibiotics [6]. Less toxic alternatives are under development for diseases that require long-term therapy such as tuberculosis.

\section{Development of Delpazolid (LCB01-0371)}

LegoChem Biosciences (Daejeon, Korea) is a company that develops effective and safe drugs using legochemistry technology, which enables the manipulation of substances by attaching and detaching compounds around scaffold-like Lego blocks. LegoChem Biosciences searches for novel candidate substances based on the concept that a good scaffold with novel blocks, based on medicinal chemistry, 
can accelerate the process of improving previous scaffolds with weak activity or have side effects. Delpazolid (code No: LCB01-0371), a derivative of oxazolidinone, is the first candidate antibiotic substance identified by LegoChem Biosciences.

Delpazolid is an antibiotic that targets Gram-positive bacteria (MRSA, VRE) including $M$. tuberculosis. It is currently undergoing a phase 2 clinical trial for oral (PO) administration and a phase 1 trial for intravenous (IV) administration to treat Gram-positive (MRSA, VRE) bacteraemia. Cyclic amidrazone blocks were applied to the key scaffold of delpazolid (Figure 1). In general, after a drug is absorbed, it must be dissolved well to ensure proper secretion. Most small molecules with suboptimal pharmacokinetic (PK) profiles tend to have low solubility. In general, small-molecule ligands that bind their targets with high efficiency are more hydrophobic, and hydrophobic interactions are essential for increased ligand efficiency [7]. Hydrophobicity not only increases target binding efficacy, but also decreases the solubility of a small molecule. The cyclic amidrazone (Figure 1) on the side chain of delpazolid maintains its hydrophobicity to some extent and has a slightly basic $\mathrm{pH}$ similar to that of carboxylate. Therefore, it can be charged by obtaining a proton from carboxylic acid under human physiological conditions, which enhances the solubility and PK profile. Therefore, the drug is accumulated slowly and excreted well, and can be administered over the long-term with minimal side effects.

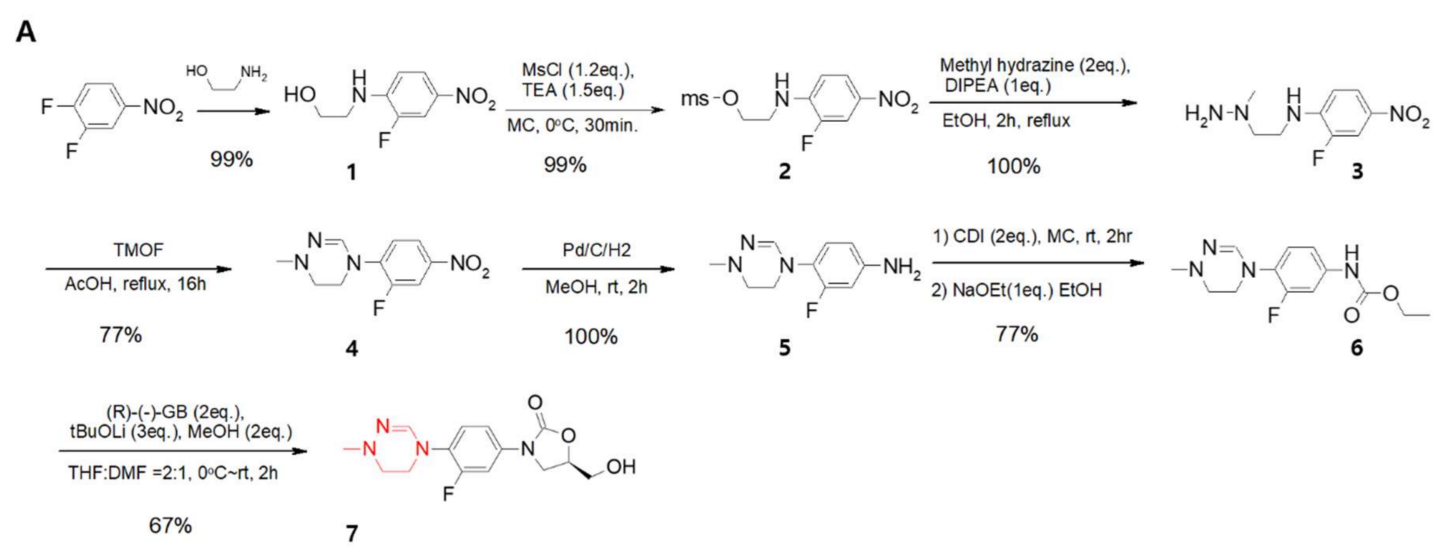

B

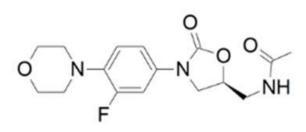

Figure 1. (A). Synthetic scheme showing that delpazolid can be synthesised in only seven steps with difluoro-nitrobenzene as the starting material. Each step shows a high yield and the products are easily purified without chromatography. The red color indicates cyclic amidrazone. (B). Chemical structure of linezolid.

\section{Safety Evaluation in the Phase 1 Clinical Trial as PO}

The greatest advantage associated with delpazolid is its safety. In phase 1a of a phase 1 clinical trial to evaluate its safety, as illustrated in Table 1, 64 subjects were divided into eight groups, six of whom were administered delpazolid and two who were administered the placebo. The study was the first double-blind, randomized human trial of delpazolid. To deliver single-ascending-doses (SADs), delpazolid was administered in a step-wise manner from $50 \mathrm{mg}$ up to $3200 \mathrm{mg}$. Only mild adverse events were observed up to $2400 \mathrm{mg}$. At a delpazolid dose of $3200 \mathrm{mg}$, gastrointestinal (GI) tract-related adverse events were noted. In the $3200 \mathrm{mg}$ dose group, volunteers had to ingest 16 tablets of $200 \mathrm{mg}$ delpazolid tablets at once, resulting in GI tract-related adverse events. Therefore, the maximum tolerated dose of delpazolid was determined to be $2400 \mathrm{mg}$ per day. 
Table 1. Summary of phase $1 \mathrm{a} / \mathrm{b}$ and $2 \mathrm{a}$ dose-escalation study to assess the safety, tolerability, and pharmacokinetics of delpazolid as a single agent.

\begin{tabular}{|c|c|}
\hline Clinical Trial Phase & Experimental Design and Adverse Effects Reported \\
\hline \multirow{4}{*}{$\begin{array}{l}\text { Phase 1a [9] } \\
\text { (SAD) }\end{array}$} & Study design: Double blind, randomized, placebo control, first-in-human design \\
\hline & $\mathrm{N}=64,8$ subject per group ( 6 active +2 placebo $)$ \\
\hline & Doses: $50,100,200,400,800,1,600,2,400$, and 3,200 mg \\
\hline & MTD: $2,400 \mathrm{mg}$ (Up to $2,400 \mathrm{mg}$, only mild adverse events were reported) \\
\hline \multirow{4}{*}{$\begin{array}{l}\text { Phase } 1 \mathrm{~b}[8] \\
\text { (MAD-7 days) }\end{array}$} & Study design: Double blind, randomized, placebo control \\
\hline & $\mathrm{N}=32,8$ subject per group ( 6 active +2 placebo $)$ \\
\hline & Doses: $400,800,1,200,1,600 \mathrm{mg}$ BID for 7 days \\
\hline & $\begin{array}{l}\text { MTD: 1,200 mg BID (Up to 2,400 mg/day, only mild adverse events were } \\
\text { reported) }\end{array}$ \\
\hline \multirow{4}{*}{$\begin{array}{c}\text { Phase } 1 \mathrm{~b} \text { [6] } \\
\text { (MAD-21 days) }\end{array}$} & Study design: Double blind, randomized, placebo control \\
\hline & $\mathrm{N}=36,12$ subject per group ( 10 active +2 placebo $)$ \\
\hline & Doses: $800 \mathrm{mg}$ QD and BID, 1,200 mg BID for 21 days \\
\hline & MTD: 1,200 mg BID (Up to 2,400mg/day, No SAE reported) \\
\hline \multirow{3}{*}{$\begin{array}{l}\text { Phase } 2 \mathrm{a}^{a} \\
\text { (EBA Trial) }\end{array}$} & Study design: Open label, randomized \\
\hline & $\begin{array}{l}\mathrm{N}=80,16 \text { subject per delpazolid group; } 8 \text { patients in active control groups, } \\
\text { HRZE and linezolid }\end{array}$ \\
\hline & $\begin{array}{l}\text { Doses: Delpazolid } 400 \mathrm{mg} \text { BID, } 800 \mathrm{mg} \text { QD, } 800 \mathrm{mg} \text { BID, 1,200 mg QD, } \\
\text { HRZE and linezolid } 600 \mathrm{mg} \text { BID for } 14 \text { days }\end{array}$ \\
\hline
\end{tabular}

Dose-escalation process consisted of a single-ascending-dose phase (SAD) and multiple-ascending-dose phase (MAD). MTD, maximum tolerated dose; $\mathrm{QD}$, quaque die (daily); BID, bis in die (twice per day); SAE, serious adverse event; EBA, early bactericidal activity; HRZE, isoniazid (H), rifampin (R), pyrazinamide (Z), and ethambutol (E). ${ }^{a}$ Results were not yet published.

A phase $1 \mathrm{~b}$ study was conducted based on multiple-ascending-doses (MADs) over seven days. Thirty-two subjects were divided into eight groups, six of whom were administered delpazolid and two of whom were administered the placebo. Subjects were given delpazolid in MADs from $400 \mathrm{mg}$ BID (bis in die, twice a day) up to $1600 \mathrm{mg}$ BID over seven days. Doses up to $1200 \mathrm{mg}$ BID for seven days were well-tolerated with no specific adverse events observed. After the 7-day MAD study, a 21-day MAD study was conducted to evaluate bone marrow toxicity, which is one of the most critical side effects of linezolid [8]. Subjects administered $800 \mathrm{mg}$ once a day (QD) to $1200 \mathrm{mg}$ BID delpazolid were monitored for up to three weeks to more accurately assess adverse events such as myelosuppression, as signs such as decreased platelet count may be observed even after two weeks. As illustrated in Table 1, serious adverse events were not observed under the MAD-21-day condition. In summary, no myelosuppression-related adverse events or serious adverse events were observed in phase $1 \mathrm{a}$ with SADs up to $2400 \mathrm{mg}$ and in phase $1 \mathrm{~b}$ with MAD up to $1200 \mathrm{mg}$ BID (2400 mg per day) for 21 days. Therefore, delpazolid does not appear to exhibit adverse events associated with repeated dosing. In addition, delpazolid did not cause CYP-mediated metabolism and cardiac repolarisation issues [6,9-11].

\section{Poor PK Profiles but Safe for Humans}

The underlying antibacterial mechanism of delpazolid is similar to that of oxazolidinone in that it inhibits bacterial protein synthesis, which kills or inhibits the growth of bacteria [12]. However, protein synthesis also occurs in the mitochondria of eukaryotes, although mitochondria use independent protein-synthesis machinery that differs from nuclear-encoded protein synthesis in the cytoplasm. In humans, 13 genes are translated into proteins through this process, all of which participate in synthesizing membrane proteins associated with oxidative phosphorylation [13]. 
However, oxazolidinones uniformly inhibit human mitochondrial protein synthesis [14]. Similarly, linezolid, an oxazolidinone analogue used to treat $\mathrm{TB}$, inhibits mitochondrial protein synthesis with potentially severe clinical consequences [15]. Therefore, the inhibition of protein synthesis by oxazolidinone intended to kill bacteria can impair mitochondria inside eukaryotic cells. Furthermore, myelosuppression may be a product of linezolid inhibition of mitochondrial protein synthesis [16].

As shown in Table 2, delpazolid showed a greater inhibitory effect than linezolid towards Escherichia coli at a 5-fold lower concentration $(0.8 \mu \mathrm{g} / \mathrm{mL})$.

Table 2. Antibiotic properties of oxazolidinones on bacterial and mitochondrial protein synthesis.

\begin{tabular}{lllll}
\hline Compound & Bacteria & Human Mitochondria (IC 50 ) & Animal Mitochondria [14] \\
\hline & Escherichia coli & K562 cell & AC16 cell & $\begin{array}{l}\text { Rat, Rabbit } \\
\text { (liver \& heart) }\end{array}$ \\
Delpazolid & $2.6 \mu \mathrm{M}$ & $4.8 \mu \mathrm{M}$ & $10.9 \mu \mathrm{M}$ & $\mathrm{NA}$ \\
& $(0.8 \mu \mathrm{g} / \mathrm{mL})$ & $(1.5 \mu \mathrm{g} / \mathrm{mL})$ & $(3.4 \mu \mathrm{g} / \mathrm{mL})$ & \\
Linezolid & $11.6 \mu \mathrm{M}$ & $3.1 \mu \mathrm{M}$ & $10.0 \mu \mathrm{M}$ & $12.8 \mu \mathrm{M}$ \\
& $(3.9 \mu \mathrm{g} / \mathrm{mL})$ & $(1.0 \mu \mathrm{g} / \mathrm{mL})$ & $(3.4 \mu \mathrm{g} / \mathrm{mL})$ & \\
\hline
\end{tabular}

In addition, in a study of human cells (immortalised myelogenous leukaemia cell line K562 and human cardiomyocyte cell line AC16), delpazolid showed inhibitory effects on mitochondrial protein synthesis similar to those of linezolid. Although delpazolid exhibited activity superior to that of linezolid in prokaryotic protein synthesis inhibition, it had similar negative effects on mitochondrial protein synthesis. Therefore, delpazolid doses lower than linezolid doses would be adequate for the treatment of Gram-positive bacteria, including TB. A lower dose would effectively inhibit bacterial protein synthesis, with relatively fewer adverse effects on human mitochondrial protein synthesis.

The association between delpazolid and myelosuppression, one of the most serious side effects of linezolid, was also tested. Healthy subjects were administered delpazolid and linezolid, and the plasma area under the concentration-time curve (AUC) was determined. As shown in Figure 2, subjects were administered delpazolid at doses ranging from 400 to $1200 \mathrm{mg}$, and $600 \mathrm{mg}$ linezolid as the comparator.

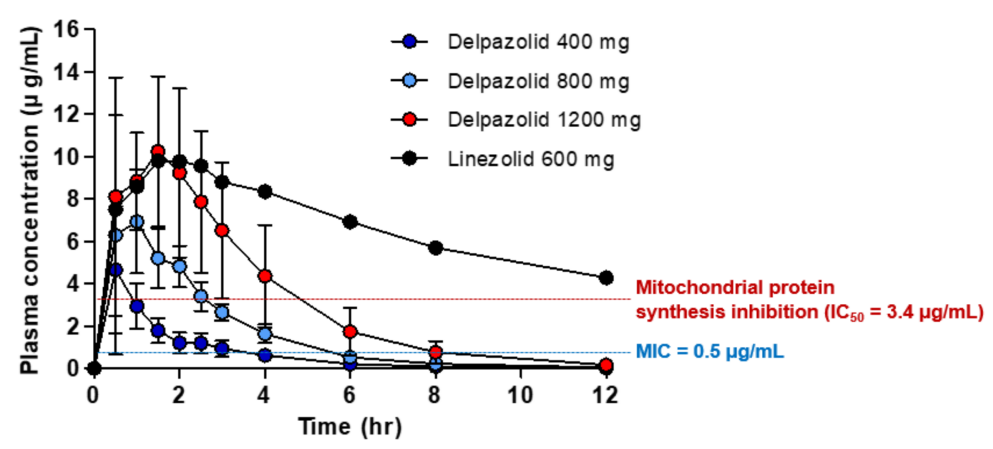

Figure 2. Mean plasma concentrations of linezolid and delpazolid in adults following oral dosing (mean \pm standard deviation, $n=6$ ).

As shown in Table 2, the $\mathrm{IC}_{50}$ values of delpazolid and linezolid at which mitochondrial protein synthesis in the two human cell-lines (K562 and AC16) were similar at $3.4 \mu \mathrm{g} / \mathrm{mL}$ indicated that these agents killed approximately $50 \%$ of human cells at $3.4 \mu \mathrm{g} / \mathrm{mL}$. Thus, $3.4 \mu \mathrm{g} / \mathrm{mL}$ of delpazolid and linezolid is the mitochondrial damage limit. At higher concentrations, mitochondrial protein synthesis is affected severely, leading to cell death. Therefore, considering $3.4 \mu \mathrm{g} / \mathrm{mL}$ as the reference value at which toxicity of the two drugs occurs, a phase 1 trial based on linezolid $600 \mathrm{mg}$ BID revealed that the linezolid plasma concentration was maintained at above the $\mathrm{IC}_{50}(3.4 \mu \mathrm{g} / \mathrm{mL})$ for $12 \mathrm{~h}$. However, delpazolid $800 \mathrm{mg}$ maintained the $\mathrm{IC}_{50}$ above the mitochondrial damage limit for only $3 \mathrm{~h}$, after which it was cleared rapidly from the blood. Therefore, delpazolid provides ample time for mitochondria to recover its protein synthesis function. In addition, increasing the delpazolid dose to $1200 \mathrm{mg}$ raises the $\mathrm{IC}_{50}$ 
to above $3.4 \mu \mathrm{g} / \mathrm{mL}$ for only $5 \mathrm{~h}$, after which it also clears from the blood. Therefore, the low AUC with rapid clearance in delpazolid ironically minimizes cellular toxicity. Consequently, repeated BID dosing of delpazolid results in much lower levels of myelosuppression because of the lower mitochondrial protein synthesis inhibition compared to linezolid $[9,10]$. Therefore, the side effects of delpazolid were much milder than those of linezolid. The difference in side effects despite the similar structure of the two drugs may be due to differences in their chemical structures. The cyclic amidrazone side chain of delpazolid facilitates more rapid clearance and prevents accumulation in the plasma compared to linezolid. Thus, rapid clearance has been demonstrated as a key advantage that reduces myelosuppression compared to linezolid. Therefore, delpazolid may replace linezolid for MDR-TB for long-term treatment [11].

\section{Toxicology}

In vivo animal toxicity tests on delpazolid did not reveal specific toxicity profiles for six months in rats and for nine months in dogs. Furthermore, genetic toxicity tests, including the Ames test, in vitro chromosomal aberration test, and rat micronucleus test, as well as pharmacological safety tests including the hERG safety test, cardiovascular, respiratory and neurobehavioral tests, and reproductive toxicity tests were conducted, none of which revealed a specific toxicity profile (Table 3).

Table 3. Toxicology summary (PO: per oral /IV: intravenous). The general toxicity of delpazolid in animals lasted up to six months in rats and nine months in dogs, and no unusual findings after long-term treatment ${ }^{a}$.

\begin{tabular}{|c|c|c|c|}
\hline General Toxicity & Status & PO & IV \\
\hline Single dose acute toxicity study in rats & Completed & $\mathrm{MTD}=2000 \mathrm{mpk}$ & $\mathrm{MTD}=1000 \mathrm{mpk}$ \\
\hline \multirow{3}{*}{$\begin{array}{l}\text { Single dose acute toxicity study in dogs } \\
4 \text { - week toxicity study in rats with } 4 \text {-week } \\
\text { recovery } \\
\text { 4- week toxicity study in dogs with } 4 \text {-week } \\
\text { recovery }\end{array}$} & Completed & $\mathrm{MTD}=1000 \mathrm{mpk}$ & $\mathrm{MTD}=500 \mathrm{mpk}$ \\
\hline & Completed & NOAEL $=60 \mathrm{mpk}$ & NOAEL $=120 \mathrm{mpk}$ \\
\hline & Completed & $\begin{array}{l}\text { NOAEL }(\text { male }=20 \mathrm{mpk} \\
\text { female }=10 \mathrm{mpk})\end{array}$ & NOAEL = $15 \mathrm{mpk}$ \\
\hline $\begin{array}{l}\text { 26- week( } 6 \text { months) toxicity study } \\
\text { in rats with } 4 \text {-week recovery }\end{array}$ & Completed & $\begin{array}{l}\text { NOAEL } \\
(\text { male }=10 \mathrm{mpk}, \\
\text { female }=100 \rightarrow 75 \mathrm{mpk})\end{array}$ & - \\
\hline $\begin{array}{l}\text { 39- week ( } 9 \text { months) toxicity study } \\
\text { in dogs with } 4 \text {-week recovery }\end{array}$ & Completed & NOAEL $=10 \mathrm{mpk}$ & - \\
\hline \multicolumn{4}{|l|}{ Genetic Toxicity } \\
\hline Ames test & Completed & Negative & \\
\hline In vitro chromosomal aberration test & Completed & Negative & \\
\hline Rat micronucleus test & Completed & Negative & \\
\hline \multicolumn{4}{|l|}{ Safety Pharmacology } \\
\hline $\begin{array}{l}\text { Assessment of blockage of hERG potassium } \\
\text { channels }\end{array}$ & Completed & Negative $\left(\mathrm{IC}_{50}>100 \mu \mathrm{M}\right)$ & \\
\hline $\begin{array}{l}\text { Cardiovascular telemetry study in beagle } \\
\text { dogs }\end{array}$ & Completed & Negative & \\
\hline Respiratory (Pulmonary) study in rats & Completed & Negative & \\
\hline Neurobehavioral safety evaluation in rats & Completed & Negative & \\
\hline Reproductive Toxicity & & PO & \\
\hline $\begin{array}{l}\text { Fertility and Embryonic Development to } \\
\text { Implantation toxicity in rat }\end{array}$ & Completed & $\begin{array}{l}\text { NOAEL }(\text { male }=15 \mathrm{mpk} \\
\text { female }=60 \mathrm{mpk})\end{array}$ & \\
\hline Embryo-Fetal Development toxicity in rat & Completed & NOAEL $=15 \mathrm{mpk}$ & \\
\hline
\end{tabular}

MTD; maximum tolerated dose, NOAEL; no-observed-adverse-effect level. ${ }^{a}$ Results were not yet published. 
In a human bioavailability study, the bioavailability of the PO form was $99-100 \%$ (800 mg) of that of the IV form. Considering that the PK profiles between the IV and PO forms are similar, conversion would be relatively easy in the future. Because delpazolid is slightly polar, it exhibits low protein binding (37\% in human), rapid clearance with no accumulation, and no food-related effects (Table 4).

Table 4. Phase 1 study: summary of delpazolid pharmacokinetic parameters. IV infusion $400 \mathrm{mg}$ and PO $800 \mathrm{mg}$, cross-over study IV administration of delpazolid was generally safe and well-tolerated $800 \mathrm{mg}$ (PO): Bioavailability was approximately 99\% switchable between the PO and IV, with no dose adjustment.

\begin{tabular}{|c|c|c|c|}
\hline $\begin{array}{l}\text { Pharmacokinetic } \\
\text { Parameter }{ }^{a}\end{array}$ & $\begin{array}{l}\text { IV Infusion; } \\
200 \text { mg }(n=6)\end{array}$ & $\begin{array}{l}\text { IV Infusion; } \\
400 \mathrm{mg}(\mathrm{n}=8)\end{array}$ & $\begin{array}{l}\text { PO; } \\
800 \text { mg }(n=8)\end{array}$ \\
\hline $\mathrm{C}_{\max }(\mu \mathrm{g} / \mathrm{mL})$ & $2.92 \pm 0.46$ & $5.25 \pm 0.96$ & $8.20 \pm 3.47$ \\
\hline $\mathrm{T}_{\max }(\mathrm{hr})$ & $0.83 \pm 0.13$ & $0.84 \pm 0.13$ & $1.22 \pm 0.98$ \\
\hline $\mathrm{T}_{1 / 2}(\mathrm{hr})$ & $1.70 \pm 0.26$ & $1.48 \pm 0.16$ & $1.64 \pm 0.48$ \\
\hline $\mathrm{AUC}_{0-24 \mathrm{~h}}(\mu \mathrm{g} \cdot \mathrm{hr} / \mathrm{mL})$ & $5.59 \pm 0.98$ & $9.39 \pm 1.46$ & $18.65 \pm 4.88$ \\
\hline $\mathrm{AUC}_{\text {inf }}(\mu \mathrm{g} \cdot \mathrm{hr} / \mathrm{mL})$ & $5.63 \pm 1.00$ & $9.42 \pm 1.47$ & $18.86 \pm 4.99$ \\
\hline $\mathrm{V}_{\mathrm{ss}}, \mathrm{V}_{\mathrm{Z}} / \mathrm{F}(\mathrm{L} / \mathrm{kg})$ & $0.90 \pm 0.06$ & $1.05 \pm 0.20$ & $1.67 \pm 0.79$ \\
\hline $\mathrm{CL}, \mathrm{Cl} / \mathrm{F}(\mathrm{L} / \mathrm{hr} / \mathrm{kg})$ & $0.56 \pm 0.10$ & $0.67 \pm 0.10$ & $0.69 \pm 0.18$ \\
\hline $\mathrm{MRT}_{\text {last }}(\mathrm{hr})$ & $1.55 \pm 0.21$ & $1.53 \pm 0.14$ & $2.87 \pm 0.92$ \\
\hline $\mathrm{C}_{\max , \operatorname{norm}}(\mu \mathrm{g} / \mathrm{mL})$ & $0.95 \pm 0.15$ & $0.85 \pm 0.16$ & $0.67 \pm 0.28$ \\
\hline $\operatorname{AUC}_{\mathrm{inf}, \text { norm }}(\mu \mathrm{g} \cdot \mathrm{hr} / \mathrm{mL})$ & $1.83 \pm 0.33$ & $1.53 \pm 0.24$ & $1.53 \pm 0.40$ \\
\hline $\mathrm{F}(\%)$ & - & - & $99.8 \pm 20.6$ \\
\hline
\end{tabular}

a Values are the means \pm standard deviation (range). $C_{\max }$, maximal drug concentration; $\mathrm{T}_{\max }$, time to reach $\mathrm{C}_{\max }$; $\mathrm{T}_{\frac{1}{2}}$, half-life; $\mathrm{AUC}_{0-24}$, area under the concentration-24-h curve; $\mathrm{AUC}_{\mathrm{inf}}$, AUC from time zero extrapolated to infinity; $\mathrm{V}_{\mathrm{ss}}$, steady-state volume of distribution; $\mathrm{V}_{\mathrm{z}} / \mathrm{F}$, apparent volume of distribution; $\mathrm{CL}$, clearance; $\mathrm{Cl} / \mathrm{F}$, apparent oral clearance; $\mathrm{MRT}_{\text {last }}$, mean residence time when the drug concentration is based on values up to and including the last measured concentration; $\mathrm{C}_{\max }$, norm, $\mathrm{C}_{\max }$ divided by dose per body weight; $\mathrm{AUC}_{\mathrm{inf}}$, norm, weight-normalised $\mathrm{AUC}_{\text {inf }} ; \mathrm{F}$, bioavailability.

\section{Activity Against TB and Combination Study of Delpazolid with Other Anti-TB Agents}

Studies of the early development of delpazolid focused on Gram-positive bacteria. The efficacy of delpazolid on Gram-positive bacteria was similar or slightly better than that of linezolid. For example, in animal studies of systemic infection [17], soft tissue infection, lung infection, and thigh infection models in mice, delpazolid showed greater efficacy than linezolid (data not shown).

To evaluate the efficacy of delpazolid in TB, an in vitro susceptibility test was conducted for M. tuberculosis H37Rv. Compared to linezolid, the minimum inhibitory concentration (MIC) for M. tuberculosis H37Rv was similar to that under delpazolid; however, the minimum bactericidal concentration was more than 4-fold lower under delpazolid (Table 5).

Table 5. Drug activities and resistance rates of linezolid and delpazolid.

\begin{tabular}{lll}
\hline Drug Activities / Resistant Rate $^{a}$ & Linezolid & Delpazolid \\
\hline MIC value for M. tuberculosis H37Rv $(\mu \mathrm{g} / \mathrm{mL})_{\text {MBC }_{99} \text { value for M. tuberculosis H37Rv }(\mu \mathrm{g} / \mathrm{mL})}$ & 0.5 & 0.5 \\
MDR-TB MIC $_{90}(\mu \mathrm{g} / \mathrm{mL})$ & 1 & 4 \\
XDR-TB MIC $_{90}(\mu \mathrm{g} / \mathrm{mL})$ & 0.25 & 0.5 \\
ECOFFs (epidemiological cutoff values) $(\mu \mathrm{g} / \mathrm{mL})$ & 1.0 & 1 \\
Resistant rate of MDR-TB $(\%)$ & 6.7 & 2.0 \\
Resistant rate of XDR-TB $(\%)$ & 4.2 & 0.8 \\
\hline
\end{tabular}

${ }^{a}$ A total of 240 M. tuberculosis isolates were tested for ECOFFS and resistant rates, including 120 MDR-TB isolates and 120 XDR-TB samples in China.

The $\mathrm{MIC}_{90}$ values of delpazolid for MDR/extensively drug resistant (XDR) TB isolates were 0.25 and $1 \mu \mathrm{g} / \mathrm{mL}$, respectively. However, an in vitro study of MDR/XDR TB isolates from China showed that the resistance rate varied considerably. The resistance of MDR-TB to linezolid was $6.7 \%$, whereas that 
to delpazolid was $0.8 \%$, suggesting higher potential efficacy of delpazolid in the treatment of MDR-TB, although no significant difference in resistance rates was observed between linezolid and delpazolid among XDR-TB isolates [18]. Therefore, delpazolid has been considered as a targeted application for MDR-TB treatment. Considering the significantly lower resistance rate of MDR-TB against delpazolid despite its similar structure to linezolid, further studies are needed to investigate structural variations in delpazolid to evaluate the correlations between the structures of various delpazolid derivatives and their resistance rates. In addition, intracellular MICs of delpazolid that can inhibit the growth of intracellular M. tuberculosis H37Rv revealed efficacy levels similar to those of linezolid under low concentrations, whereas delpazolid had greater efficacy at higher concentrations (Figure 3).

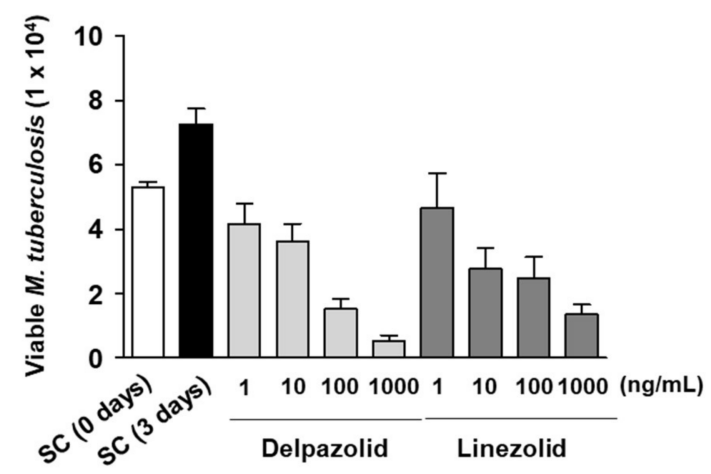

Figure 3. Intracellular activity of delpazolid. The activity of delpazolid on intracellular M. tuberculosis was compared to linezolid in bone marrow-derived macrophages (BMDMs) at three days after infection. The experiment was performed in triplicate, and the results are shown as the mean \pm standard error of the mean (SEM). SC, solvent control.

The treatment of tuberculosis requires a combination of several antimicrobial agents and long-term therapy [19]. Therefore, evaluating synergy with other anti-TB agents is a crucial step in finding drugs that can be co-administered with delpazolid. As indicated in Table 6, a checkerboard assay was performed to identify pre-existing anti-TB medications with potential synergistic effects with delpazolid.

Table 6. MICs of selected anti-tuberculosis compounds against M. tuberculosis H37Rv and corresponding interaction profiles with delpazolid assessed by checkerboard.

\begin{tabular}{|c|c|c|c|c|c|}
\hline Ref. Drug & $\operatorname{MIC}(\mu \mathrm{g} / \mathrm{mL})$ & Tested TB Drugs & $\operatorname{MIC}(\mu \mathrm{g} / \mathrm{mL})$ & FIC index & Activity $^{a}$ \\
\hline \multirow{16}{*}{ Delpazolid } & \multirow{16}{*}{1} & Isoniazid & 0.13 & 1.13 & I \\
\hline & & Rifampicin & 0.06 & 0.75 & Ad \\
\hline & & Rifapentine & 0.01 & 0.75 & $\mathrm{Ad}$ \\
\hline & & Ethambutol & 0.50 & 1.02 & I \\
\hline & & Cycloserine & 4.0 & 1.02 & I \\
\hline & & Amikacin & 0.04 & 1.02 & I \\
\hline & & Streptomycin & 0.25 & 1.02 & I \\
\hline & & Capreomycin & 0.31 & 1.02 & I \\
\hline & & Moxifloxacin & 0.06 & 0.75 & Ad \\
\hline & & Levofloxacin & 0.25 & 0.75 & Ad \\
\hline & & Clofazimine & 0.25 & 0.52 & pS \\
\hline & & Bedaquiline & 0.25 & 0.53 & pS \\
\hline & & Delamanid & 0.02 & 0.75 & Ad \\
\hline & & Ethionamide & 0.5 & 1.03 & I \\
\hline & & $p$-aminosalicylic acid & 0.02 & 1 & I \\
\hline & & Pyrazinamide $\mathrm{b}$ & 200 & 0.63 & $\mathrm{pS}$ \\
\hline
\end{tabular}

a S: synergy, pS: partial synergy, Ad: additive, I: indifference. ${ }^{\mathrm{b}}$ Tested in acidic condition (pH 5.2) 
The assay revealed that delpazolid has partial synergism with clofazimine, bedaquiline, and pyrazinamide. Based on the results, in vitro time-kill kinetics tests were conducted by combining delpazolid with clofazimine and bedaquiline (Figure 4).
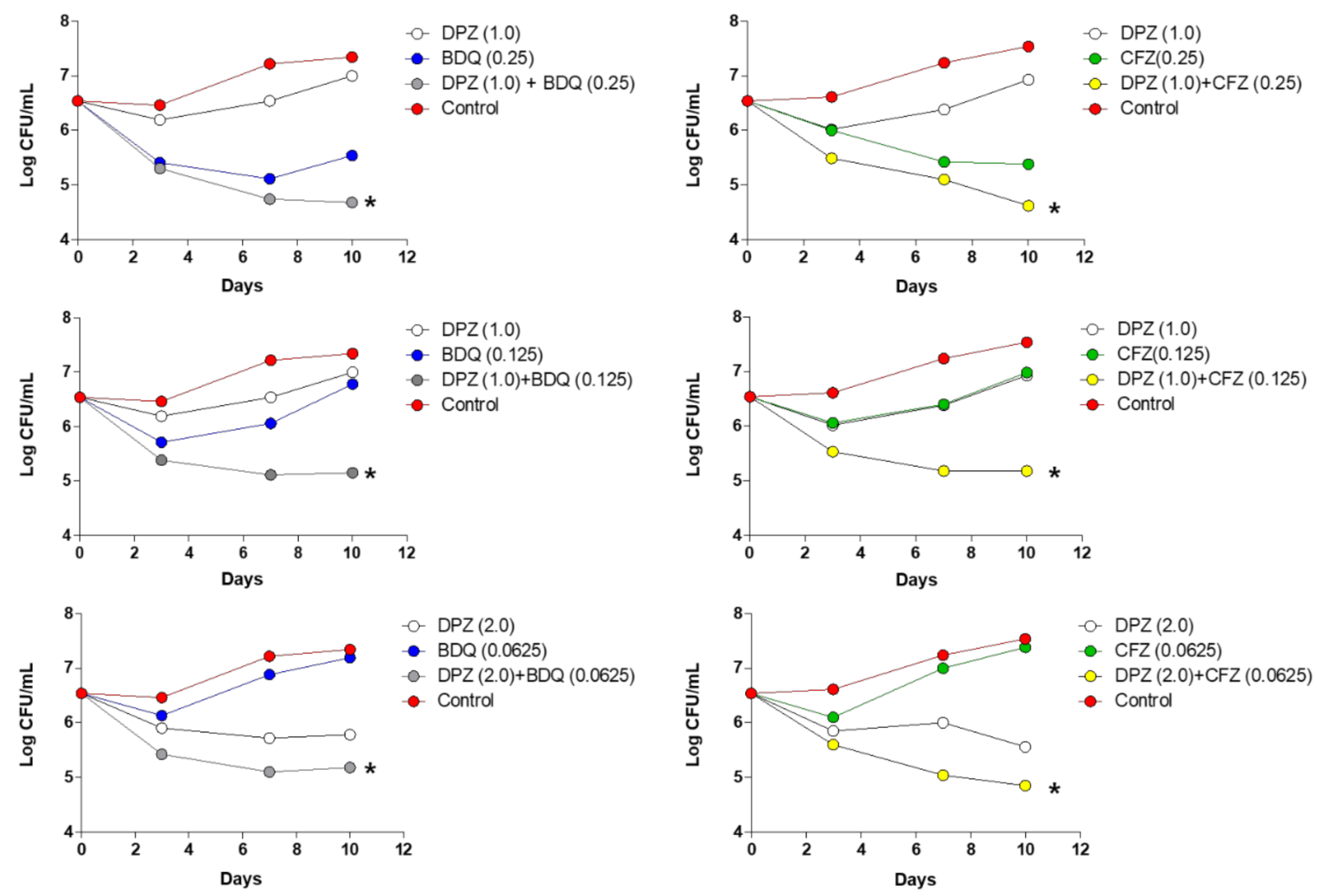

Figure 4. In vitro combination time-kill assay with anti-TB drugs. Viability of $M$. tuberculosis $\mathrm{H} 37 \mathrm{Rv}$ was evaluated using combinations of various concentrations of delpazolid and bedaquiline or clofazimine $(\mu \mathrm{g} / \mathrm{mL})$.

Using the MIC against M. tuberculosis H37Rv for each drug, changes in colony-forming units (CFU) with monotherapy or combination therapy were evaluated. In addition, based on the MICs, synergistic effects between delpazolid plus bedaquiline and delpazolid plus clofazimine were evaluated at varying doses. Although the CFUs decreased at the MIC of a single drug, regrowth was observed over time. However, when delpazolid was combined with bedaquiline or clofazimine, using the $0.5 \times$ MIC of each drug, no regrowth was observed (Figure 4). In addition, the combination of bedaquiline and clofazimine with delpazolid consistently suppressed the growth of $M$. tuberculosis H37Rv, exhibiting high synergistic effects with $1 \times$ MIC delpazolid $(1 \mu \mathrm{g} / \mathrm{mL})$ and $0.5 \times$ MIC clofazimine $(0.25 \mu \mathrm{g} / \mathrm{mL})$, resulting in a $2 \log \mathrm{CFU}$ reduction in M. tuberculosis H37Rv. Synergy between two new antimycobacterial compounds, such as delpazolid and bedaquiline or clofazimine, offers an attractive foundation for a new tuberculosis regimen.

\section{Activity of Delpazolid on Nontuberculous Mycobacteria}

Nontuberculous mycobacteria (NTM) are naturally occurring organisms found in water and soil. They are associated with biofilm formation, which enhances their disinfectant and antibiotic resistance. Particularly, Mycobacterium avium complex and Mycobacterium abscessus are the most common causes of pulmonary NTM and deadly pathogens, with high failure rates and relapse rates that may exceed $40 \%$ [20]. Although most people are not affected by such pathogens, in some individuals susceptible to conditions such as cystic fibrosis, chronic obstructive lung disease, bronchiectasis, and thoracic skeletal abnormalities, progressive and debilitating disease can occur [21]. 
A key concern in NTM treatment is the lack of antibiotics appropriate for long-term treatment for diverse NTM pathogens. Here, we evaluated the activity of delpazolid via in vitro susceptibility tests, as shown in the table below (Table 7) [22].

Table 7. MICs of antibiotics against clinical isolates of NTMs.

\begin{tabular}{|c|c|c|c|c|}
\hline $\begin{array}{l}\text { NTM Species } \\
\text { (no. of strain tested) }\end{array}$ & Antibiotics & $\begin{array}{l}\text { Range } \\
(\mu \mathrm{g} / \mathrm{mL})\end{array}$ & $\begin{array}{l}\mathrm{MIC}_{50} \\
(\mu \mathrm{g} / \mathrm{mL})\end{array}$ & $\begin{array}{l}\text { MIC }_{90} \\
(\mu \mathrm{g} / \mathrm{mL})\end{array}$ \\
\hline \multirow{3}{*}{ Mycobacterium avium (22) } & Delpazolid & $8-0.125$ & 2 & 8 \\
\hline & Linezolid & $8-0.125$ & 2 & 8 \\
\hline & Clarithromycin & $>128-\leq 0.125$ & $>128$ & $>128$ \\
\hline \multirow{3}{*}{$\begin{array}{c}\text { Mycobacterium abscessus } \\
(20)\end{array}$} & Delpazolid & $8-0.25$ & 2 & 8 \\
\hline & Linezolid & $16-0.5$ & 4 & 8 \\
\hline & Clarithromycin & $128-\leq 0.125$ & $\leq 0.125$ & 1 \\
\hline \multirow{3}{*}{$\begin{array}{l}\text { Mycobacterium fortuitum } \\
\text { (21) }\end{array}$} & Delpazolid & $2-0.25$ & 1 & 2 \\
\hline & Linezolid & $8-0.5$ & 2 & 8 \\
\hline & Clarithromycin & $8-\leq 0.125$ & 0.25 & 4 \\
\hline \multirow{3}{*}{$\begin{array}{c}\text { Mycobacterium kansasii } \\
\text { (22) }\end{array}$} & Delpazolid & $2-0.25$ & 1 & 2 \\
\hline & Linezolid & $2-0.25$ & 0.5 & 2 \\
\hline & Clarithromycin & $0.125-\leq 0.125$ & $\leq 0.125$ & $\leq 0.125$ \\
\hline \multirow{3}{*}{$\begin{array}{l}\text { Mycobacterium chelonae } \\
(20)\end{array}$} & Delpazolid & $4-0.25$ & 1 & 2 \\
\hline & Linezolid & $8-0.5$ & 2 & 4 \\
\hline & Clarithromycin & $0.2-\leq 0.025$ & 0.1 & 0.2 \\
\hline
\end{tabular}

Delpazolid had MICs similar to those of linezolid against M. avium, M. abscessus, M. fortuitum, M. kansasii, and M. chelonae, and inhibited NTM proliferation. In particular, delpazolid was effective against several $M$. abscessus strains in vitro and in a macrophage infection model. Acute infections in C57BL/6 mice, delpazolid $100 \mathrm{mg} / \mathrm{kg}$ exhibited greater in vivo efficacy than clarithromycin $200 \mathrm{mg} / \mathrm{kg}$, a macrolide that is the main drug currently for M. abscessus treatment [12]. Therefore, delpazolid represents a promising novel class of oxazolidinones with improved safety for the treatment of $M$. abscessus.

\section{Conclusions}

As observed in clinical studies, the greatest advantage of delpazolid over linezolid is the potential for delpazolid to be used in long-term therapies. The development of delpazolid has focused on TB treatment, as this disease requires long-term treatment. In December 2016, LegoChem Biosciences entered into a license agreement with RMX Biopharma for the development, manufacture, and commercialization of delpazolid in China. In addition, delpazolid received an FDA orphan drug designation, a Qualified Infectious Disease Product Designation, and was selected as a Fast Track target drug.

On October 30, 2019, at 'The 50th Union World Conference on Lung Health,' held in Hyderabad, India, LegoChem Biosciences released the interim efficacy and safety results of a phase 2a study on delpazolid. Particularly, the results of a clinical phase 2 a early bactericidal activity trial involving 79 Korean patients with drug-susceptible tuberculosis were reported. The findings will be further analyzed to determine the doses appropriate for different patient populations to guide further phase 2a studies. The phase 1 trial revealed that myelosuppression can be reduced, and phase 2a results suggested that delpazolid can replace linezolid as a therapy for TB and reduce the treatment period.

Author Contributions: Conceptualization, Y.L.C. and J.J.; writing, J.J.; review and editing, Y.L.C. and J.J.; visualization, Y.L.C. and J.J.; project administration, Y.L.C. and J.J. All authors have read and agreed to the published version of the manuscript.

Acknowledgments: We thank all those who provided important information that was part of this work. We also thank Tae ho Kim for his assistance in figure preparation. 
Conflicts of Interest: The authors declare no conflict of interest.

\section{References}

1. Bozdogan, B.; Appelbaum, P.C. Oxazolidinones: Activity, mode of action, and mechanism of resistance. Int J Antimicrob Agents. 2004, 23, 113-119. [CrossRef] [PubMed]

2. Marchese, A.; Schito, G.C. The oxazolidinones as a new family of antimicrobial agent. Clin Microbiol Infect. 2001, 4, 66-74. [CrossRef] [PubMed]

3. Moellering, R.C. Linezolid: The first oxazolidinone antimicrobial. Ann Intern Med. 2003, 138, $135-142$. [CrossRef] [PubMed]

4. Millard, J.; Pertinez, H.; Bonnett, L.; Hodel, E.M.; Dartois, V.; Johnson, J.L.; Caws, M.; Tiberi, S.; Bolhuis, M.; Alffenaar, J.C.; et al. Linezolid pharmacokinetics in MDR-TB: A systematic review, meta-analysis and Monte Carlo simulation. J Antimicrob Chemother. 2018, 73, 1755-1762. [CrossRef]

5. Gerson, S.L.; Kaplan, S.L.; Bruss, J.B.; Le, V.; Arellano, F.M.; Hafkin, B.; Kuter, D.J. Hematologic effects of linezolid: Summary of clinical experience. Antimicrob Agents Chemother. 2002, 46, 2723-2726. [CrossRef]

6. Choi, Y.; Lee, S.W.; Kim, A.; Jang, K.; Nam, H.; Cho, Y.L.; Yu, K.S.; Jang, I.J. Chung, J.Y. Safety, tolerability and pharmacokinetics of 21 day multiple oral administration of a new oxazolidinone antibiotic, LCB01-0371, in healthy male subjects. J Antimicrob Chemother. 2018, 73, 183-190. [CrossRef]

7. De Freitas Ferreira, R.; Schapira, M. A systematic analysis of atomic protein-ligand interactions in the PDB. Medchemcomm. 2017, 8, 1970-1981. [CrossRef]

8. Singh, B.; Cocker, D.; Ryan, H.; Sloan, D.J. Linezolid for drug-resistant pulmonary tuberculosis. Cochrane Database Syst Rev. 2019, 3, CD012836. [CrossRef]

9. Cho, Y.S.; Lim, H.S.; Cho, Y.L.; Nam, H.S.; Bae, K.S. Multiple-dose Safety, Tolerability, Pharmacokinetics, and Pharmacodynamics of Oral LCB01-0371 in Healthy Male Volunteers. Clin Ther. 2018, 40, 2050-2064. [CrossRef]

10. Cho, Y.S.; Lim, H.S.; Lee, S.H.; Cho, Y.L.; Nam, H.S.; Bae, K.S. Pharmacokinetics, Pharmacodynamics, and Tolerability of Single-Dose Oral LCB01-0371, a Novel Oxazolidinone with Broad-Spectrum Activity, in Healthy Volunteers. Antimicrob Agents Chemother. 2018, 62, e00451-18. [CrossRef]

11. Sunwoo, J.; Kim, Y.K.; Choi, Y.; Yu, K.S.; Nam, H.; Cho, Y.L.; Yoon, S.; Chung, J.Y. Effect of food on the pharmacokinetic characteristics of a single oral dose of LCB01-0371, a novel oxazolidinone antibiotic. Drug Des Devel Ther. 2018, 12, 1707-1714. [CrossRef] [PubMed]

12. Kim, T.S.; Choe, J.H.; Kim, Y.J.; Yang, C.S.; Kwon, H.J.; Jeong, J.; Kim, G.; Park, D.E.; Jo, E.K.; Cho, Y.L.; et al. Activity of LCB01-0371, a Novel Oxazolidinone, against Mycobacterium abscessus. Antimicrob Agents Chemother. 2017, 61, e02752-16. [CrossRef] [PubMed]

13. Hällberg, B.M.; Larsson, N.G. Making proteins in the powerhouse. Cell Metab. 2014, 20, 226-240. [CrossRef] [PubMed]

14. McKee, E.E.; Ferguson, M.; Bentley, A.T.; Marks, T.A. Inhibition of mammalian mitochondrial protein synthesis by oxazolidinones. Antimicrob Agents Chemother. 2006, 50, 2042-2049. [CrossRef] [PubMed]

15. De Vriese, A.S.; Coster, R.V.; Smet, J.; Seneca, S.; Lovering, A.; Van Haute, L.L.; Vanopdenbosch, L.J.; Martin, J.J.; Groote, C.C.; Vandecasteele, S.; et al. Linezolid-induced inhibition of mitochondrial protein synthesis. Clin Infect Dis. 2006, 42, 1111-1117. [CrossRef]

16. Nagiec, E.E.; Wu, L.; Swaney, S.M.; Chosay, J.G.; Ross, D.E.; Brieland, J.K.; Leach, K.L. Oxazolidinones inhibit cellular proliferation via inhibition of mitochondrial protein synthesis. Antimicrob Agents Chemother. 2005, 49, 3896-3902. [CrossRef]

17. Jeong, J.W.; Jung, S.J.; Lee, H.H.; Kim, Y.Z.; Park, T.K.; Cho, Y.L.; Chae, S.E.; Baek, S.Y.; Woo, S.H.; Lee, H.S.; et al. In vitro and in vivo activities of LCB01-0371, a new oxazolidinone. Antimicrob Agents Chemother. 2010, 54, 5359-5362. [CrossRef]

18. Zong, Z.; Jing, W.; Shi, J.; Wen, S.; Zhang, T.; Huo, F.; Shang, Y.; Liang, Q.; Huang, H.; Pang, Y. Comparison of In Vitro Activity and MIC Distributions between the Novel Oxazolidinone Delpazolid and Linezolid against Multidrug-Resistant and Extensively Drug-Resistant Mycobacterium tuberculosis in China. Antimicrob Agents Chemother. 2018, 62, e00165-18. [CrossRef]

19. Kerantzas, C.A.; Jacobs, W.R., Jr. Origins of Combination Therapy for Tuberculosis: Lessons for Future Antimicrobial Development and Application. mBio. 2017, 8, e01586-16. [CrossRef] 
20. Abate, G.; Hamzabegovic, F.; Eickhoff, C.S.; Hoft, D.F. BCG Vaccination Induces M. avium and M. abscessus Cross-Protective Immunity. Front Immunol. 2019, 10, 234. [CrossRef]

21. Johnson, M.M.; Odell, J.A. Nontuberculous mycobacterial pulmonary infections. J Thorac Dis. 2014, 6, 210-220. [PubMed]

22. DeStefano, M.S.; Shoen, C.M.; Sklaney, M.R.; Cynamon, M.H. The In Vitro Activity of Delpazolid (LCB01-0371) against Several Non-Tuberculous Mycobacteria (NTM); (Poster Number AAR-731, Friday, June 21, 2019); ASM Microbe: San Francisco, CA, USA, 2019. article distributed under the terms and conditions of the Creative Commons Attribution (CC BY) license (http://creativecommons.org/licenses/by/4.0/). 